\title{
Transparencia en la Gestión Municipal de los Barrios: Una perspectiva ciudadana
}

\author{
Neigbourhoods Municipal Management \\ Transparency, a Citizens' point of view
}

Transparência na Gestão Municipal dos Bairros: Uma perspectiva cidadã

\author{
Tamara Ortega Uribe* \\ Tomás Marín Troncoso* \\ Ciudad Viva, Centro de Urbanismo Ciudadano \\ Chile
}

\section{Resumen}

El artículo presenta los resultados de un proceso de investigación participativa para la acción de diferentes grupos ciudadanos -coordinados por Ciudad Viva- preocupados por la profundización democrática en nuestro país. Para esto, utilizamos la Ley de Transparencia como una herramienta eficaz para fortalecer capacidades de fiscalización ciudadana en el terreno de la gestión municipal de los barrios. El estudio contiene elementos de capacitación recíproca, auto investigación, revisión bibliográfica, entrevistas a actores relevantes, aplicación práctica de la ley con solicitudes, seguimiento y análisis de esta experiencia. A partir de esto, se generan propuestas, acciones e indicadores para mejorar el acceso a información en materias que debe gestionar la administración pública, especialmente municipal, pero también con fiscalización y compromiso ciudadano.

Palabras claves: acceso a la información pública, fiscalización ciudadana, gestión municipal, investigación participativa para la acción, agenda ciudadana.

\section{Abstract}

The article presents the results of a process of participatory action research of different citizen groups -coordinated by Ciudad Viva- concerned about the deepening of de-

\footnotetext{
* Tamara Ortega Uribe: Socióloga, Universidad de Valparaíso. Encargada de Transparencia y Comunidades en Ciudad Viva.<tamara@ciudadviva.cl>

* Tomás Marín Troncoso, Sociólogo, Universidad de Chile. Diplomado en Políticas Públicas, Pontificia Universidad Católica de Chile. Co- Director Ejecutivo, área de Investigación y Comunicaciones. CiudadViva. <tmarint@gmail.com>
} 
mocracy in our country. We used the Transparency Law for this, as an effective tool to strengthen citizen control capabilities in the field of municipal neighborhood management. The research contains elements of mutual-training, self-investigation, literature review, interviews with relevant stakeholders, practical application of the law with formal requests, monitoring and analysis of this experience. From this, we generated proposals, actions and indicators to improve access to information on matters to be handled by the government, especially at municipal level, but also with citizen commitment and control.

Keywords: Access to Public Information. Citizen Control. Municipal Management. Participatory Action Research. Citizen Agenda.

\section{Resumo}

O artigo apresenta os resultados de um processo de pesquisa participativa para a ação de diferentes grupos cidadãos - coordenada pelo Ciudad Viva (Cidade Viva) preocupados pelo aprofundamento democrático em nosso país. Para isso, utilizamos a Lei de Transparência como uma ferramenta eficaz para fortalecer capacidades de fiscalização cidadã no terreno da gestão municipal dos bairros. A pesquisa contém elementos de capacitação recíproca, autopesquisa, revisão bibliográfica, entrevistas a atores relevantes, aplicação prática da Lei com pedidos, acompanhamento e análise dessa experiência. A partir disso, são geradas propostas, ações e indicadores para melhorar o acesso à informação em matérias que deve gerar a administração pública, especialmente municipal, mas também com fiscalização e compromisso cidadão.

Palavras-chave: Aceso à Informação Pública. Fiscalização cidadã. Gestão Municipal. Pesquisa participativa para a ação. Agenda Cidadã.

El presente trabajo no hubiera sido posible sin el equipo ciudadano que lo fue empujando día a día. Entre ellos destacan especialmente Gerardo Lanzarotti, André Ammon, Víctor Hugo Luzzi, Jessica Gjuranovic y Juan Eduardo Donoso del Barrio Bellavista;Tusy Urra, Milsa Arancibia, Francisca Licarayen y Jaime Díaz de la Villa Olímpica;Alicia Gálvez, Francisco Osorio, Paulina Vivanco y Guillermo Hinzpeter del CerroYungay en Valparaíso; José Osorio y Claudia Manzur del Barrio Yungay; Josefa Errázuriz de la Unión Comunal de Juntas de Vecinos de Providencia y Gastón Lux de Ciudadanos por Valparaíso. Agradecemos también el apoyo de la Fundación Pro Acceso y de la Clínica Jurídica de la Universidad Alberto Hurtado.

Mención aparte merece Juan Pablo Frick, encargado de mapeo participativo, quien fue el tercer miembro del equipo motor. Apoyaron también Magdalena Morel, Diego Mallea,Aude Cranois y Lake Sagaris, todos miembros de Ciudad Viva. 


\section{Introducción: investigaciones ciudadanas para la acción}

El presente estudio se enmarca en el desarrollo de una serie de investigaciones ciudadanas llevadas a cabo por Ciudad Viva, agrupación comunitaria de base del Barrio Bellavista, con el objetivo de construir una agenda ciudadana para ciudades más justas, sustentables e inclusivas.

Refleja los anhelos de diversas comunidades por construir los cambios necesarios para avanzar hacia una sociedad más democrática y un modelo de desarrollo sostenible, a partir de una nueva manera de concebir y gestionar las ciudades y los territorios. Con ella, buscamos que el llamado "tercer sector" o ciudadanía organizada haga sus propias propuestas programáticas al mundo político y la opinión pública, en relación a lo que queremos para tener ciudades más amables, justas, seguras y sanas en el siglo $\mathrm{XXI}$.

Desde el retorno de la democracia, diversos grupos ciudadanos comenzaron a aparecer con un nuevo tipo de demandas, especialmente vinculadas al desarrollo urbano de las ciudades y el impacto que este tiene en la vida de sus habitantes. Prácticamente todas son un llamado a repensar nuestro modelo democrático, en términos de cómo hacemos partícipe a la ciudadanía en las políticas públicas destinadas a satisfacer sus demandas y necesidades. Esto es, cómo integramos a los sujetos que son objeto de la política, en el desarrollo de la política misma. La respuesta hasta el momento no ha sido satisfactoria, por lo que el número de conflictos urbanos y las organizaciones que de allí se generan va en aumento.

En este camino, una de las reformas democratizadoras más importantes de nuestro sistema político corresponde a la Ley 20.285 sobre transparencia de la función pública y acceso a la información de la administración del Estado (en adelante Ley de Transparencia), promulgada en agosto 2008, que consagra el derecho de cualquier ciudadano o ciudadana a acceder a prácticamente toda la información generada por el Estado. Esta ley constituye un avance tremendo para nuestra democracia, que debe ser defendido y potenciado.

En el ámbito del desarrollo y gestión de nuestras ciudades, la Ley de Transparencia se ha transformado en una herramienta muy importante para las agrupaciones de la sociedad civil, que les permite fiscalizar debidamente el trabajo de los servidores públicos electos por la ciudadanía. Sin embargo, todavía adolece de variados problemas en su implementación, sobre todo a nivel municipal y de los barrios.

Hoy en día, vecinos y vecinas a lo largo de todo el país enfrentan dificultades comunes en los sectores donde residen: construcción de edificios que no son armónicos con el lugar, ruidos molestos, presencia excesiva de locales con patentes de alcohol, estacionamientos ilegales, vandalismo, microbasurales, aceras en mal estado y, en general, asuntos puntuales que afectan su calidad de vida.Van destruyendo la relación comunitaria, al obligar a vecinos y vecinas a abandonar el espacio público y la vida en comunidad, replegándose en sus casas. Generan así un círculo vicioso que va destruyendo a los barrios patrimoniales, no sólo en Santiago, sino en todo Chile. 
En esta esfera, la principal contraparte, la "primera cara" del Estado son los municipios, la entidad pública más cercana a los vecinos y vecinas. Sin embargo, la puesta en marcha de la Ley de Transparencia, en general privilegió a las distintas divisiones del Gobierno Central (no funciona para el Congreso) y fue mucho más débil en su apoyo a las municipalidades. En esta ocasión, se repitió en patrón típico en la administración pública chilena: a las municipalidades se les exigió al mismo nivel que al resto, pero no se les entregó el apoyo ni las herramientas suficientes para su correcta puesta en marcha. En ocasiones también, detectamos malas prácticas injustificables de los municipios, negando la información a los y las solicitantes.

Por todo esto, como organización ciudadana decidimos llevar a cabo un proceso de investigación participativa para la acción, que nos permitiera identificar los principales problemas que afectan a los grupos de la sociedad civil, especialmente aquellas vecinales o con un arraigo territorial claro, a la hora de obtener información pública a nivel municipal y demás relacionados a la gestión urbana.

Como su nombre lo dice, este tipo de estudio no obedece a las formas típicas de hacer investigación desde un centro de estudios que, por lo general, se vinculan a un determinado paradigma: cuantitativo o cualitativo. Este proceso investigativo más bien se sitúa en el paradigma emergente de la investigación-acción y el aprender-haciendo. Retoma los planteamientos de Paulo Freire en el ámbito educativo que cuestionan la separación entre sujeto investigador (o educador) y objeto investigado (o educado).

De esta manera, los resultados del trabajo que se entregan a continuación, se instalan en un giro epistemológico para producir un sujeto investigador que es al mismo tiempo su propio investigador: las organizaciones ciudadanas leyéndose y aprendiendo de sí mismas. Buscamos así generar una capacidad de (auto) investigación ciudadana, cuyos resultados sean un llamado a la acción. En este caso, una serie de propuestas concretas para mejorar la transparencia a nivel municipal en la gestión de los barrios.

\section{Una Agenda Ciudadana para la transparencia municipal}

La Agenda Ciudadana es una iniciativa del Consejo de Ciudadanía Activa, un grupo de trabajo integrado por diversas organizaciones ciudadanas que trabajan en los temas de democratización de la gestión urbana, defensa y promoción de nuestro patrimonio, promoción del reciclaje y por un transporte sostenible y más equitativo.' Por lo tanto, se fundamenta tanto en los conocimientos actuales del mundo técnico y académico - para lo cual se basa en investigaciones realizadas por expertos académicos en los distintos temas-, como en la experiencia práctica de comunidades

I El Consejo está compuesto por el Centro Cultural el Sitio de Yungay, Ciudadanos por Valparaíso, Junta de Vecinos 35 “Bellavista”, Agrupación de Recolectores Ecológicos de El Bosque, Ecovirtual (Tomé), Junta de Vecinos 13 “Mario Baeza” Bellavista, Comunactiva (Conchali), Cooperativa Territorio Sur (Valparaíso), Junta de Vecinos 34 “Andrés BeIlo" Patronato, Mujeres Arriba de la Cleta y CiudadViva, quien ejerce la coordinación. 
organizadas a lo largo de Chile, que son quienes dirigen el proceso. Se trata, en definitiva, de un intento serio por consolidar una corriente de pensamiento y trabajo que pone al mismo nivel los conocimientos académicos y técnicos de la academia, con la experiencia concreta de las organizaciones de la sociedad civil.

No se trata de una declaración general de principios, sino de una agenda de trabajo, con propuestas concretas desde la ciudadanía, donde nos comprometemos como comunidades e individuos a realizar cambios propios, al tiempo que establecemos metas concretas en cuanto a reformas que exigiremos a nuestras autoridades locales, regionales y nacionales.

En el proceso de construcción de la agenda han sido esenciales -y seguirán siéndolo en los próximos meses- encuentros y actividades de capacitación que ofrecen la posibilidad de discutir, con altura de miras y una buena investigación y preparación, y consensuar posiciones clave sobre diversas políticas nacionales de relevancia para la gestión local. Este trabajo se realizó coordinadamente con barrios que son líderes en la gestión de sus territorios, los cuales permanentemente desarrollan acciones de incidencia en la gestión democrática de sus barrios.

Aquí subyace el corazón de los procesos de investigación participativa, pues se promueve un diálogo amplio y diverso, con representantes de variadas agrupaciones ciudadanas, el cual, además de representar un intercambio de visiones y experiencias, coloca al mando del proceso investigativo a los mismos sujetos de la investigación, con un llamado a la acción mediante el trabajo concreto de la creación de la agenda.

Por último, debemos señalar que la agenda es un programa de trabajo que exigirá la ciudadanía a las autoridades, con su primera prueba en las elecciones municipales del 2012. Es más: servirá de base para evaluar las propuestas de los candidatos a concejales y alcaldes, y posteriormente, elaborar indicadores y parámetros para evaluar los avances hacia el logro de estos objetivos. Así, buscamos promover una cultura cívica de la transparencia, produciendo información sobre los principales obstáculos y necesidades que enfrenta la ciudadanía, articulándolos con el uso, y posibles mejoras, de la Ley de Transparencia.

\section{I.Abordaje metodológico}

Como ya hemos mencionado, la investigación se desarrolló desde un enfoque que comprende dos ejes esenciales en la construcción de conocimiento: primero, que todo grupo humano tiene la capacidad de (auto) investigación ciudadana, que contribuye a dar forma y transmitir los conocimientos existentes, para movilizarlos e integrarlos en estrategias de construcción ciudadana más eficaces, democráticas, de control y fiscalización de la gestión urbana. Segundo que se trata de un estudio que tiene un fin propositivo y activo por parte de la comunidad, es decir, busca generar información respecto de la transparencia a nivel municipal y articular propuestas de trabajo coordinado entre las mismas colectividades participantes, para mejorar la transparencia y el acceso a la información en el ámbito de la gestión urbana. 
El estudio se realizó entre junio 2010 y enero $201 \mathrm{I}$. Se sustentó básicamente, aunque no de manera exclusiva, en cuatro comunidades territoriales: los barrios Bellavista,Villa Olímpica y Yungay en Santiago, y el Cerro Yungay en Valparaíso. El desarrollo de la investigación fue coordinado por los dirigentes y equipos profesionales de Ciudad Viva, pero cada territorio fue organizado por sus propias organizaciones de base: las tres juntas de vecinos de Bellavista, la Asamblea de Vecinos por la Reconstrucción de la Villa Olímpica, los Vecinos por la Defensa del Barrio Yungay y la Junta de Vecinos del Cerro Yungay. Estas organizaciones manifestaron su interés por participar en el estudio, establecieron talleres vecinales en sus sectores y permanentemente fueron comentando y modificando su progreso. Contamos, además, con el apoyo jurídico de la Fundación Pro Acceso y de la Clínica Jurídica de la Universidad Alberto Hurtado, para la aplicación práctica de la Ley de Transparencia.

En el ámbito metodológico, recurrimos a diferentes maneras de generación de información, de acuerdo a los deseos y las posibilidades de cada grupo.

Comenzamos con la revisión de antecedentes bibliográficos sobre transparencia y acceso a la información en Chile y el mundo, y su relación con las organizaciones de la sociedad civil. Adicionalmente realizamos entrevistas a informantes-actores claves, tanto de organizaciones profesionales especializadas como de vecinos activos, que día a día enfrentan problemas de transparencia en la gestión de sus barrios. De esta manera pudimos hacernos una primera idea de la situación del acceso a la información pública y su relación con los problemas vecinales y el ámbito municipal.

Posteriormente, realizamos un taller de aprendizaje sobre la Ley de Transparencia, en la que participaron representantes de los distintos grupos, así como de otros sectores y grupos cercanos (La Vega, Unión Comunal Providencia, Ciudadanos por Valparaíso, etc.). Dicho taller fue dictado por los abogados de Pro Acceso y en él los dirigentes y miembros del equipo pudimos profundizar más en los alcances y la aplicación de la ley. En este punto realizamos las primeras solicitudes efectivas, las que monitorearíamos a lo largo del proceso.

De esta fase inicial, pudimos hacer un primer set de propuestas para una agenda de reformas ciudadanas a la gestión barrial de los municipios, especialmente a nivel de transparencia y acceso a la información. Dichas propuesta fueron entonces presentadas ante una Comisión de Transparencia, integrada por dirigentes y representantes de organizaciones ciudadanas de distintos sectores de Santiago y también de otras ciudades del país (ver gráficos I y 2). ${ }^{2}$ Este proceso se desarrolló en el marco de un Encuentro Nacional de Ciudadanía Activa (agosto, 2010), en el que se realizaron distintas actividades de debate, capacitación y manifestación con diversos grupos de la ciudadanía organizada 
que se congregaron en la capital para aportar a la Agenda Ciudadana.Varias otras comisiones se refirieron a otras temáticas y los resultados del trabajo de cada una fueron luego presentados y consensuados en un plenario central, donde se discutieron las propuestas, para avanzar en la construcción de propuestas de corto, mediano y largo plazo para la Agenda.

Contando ya con un primer diagnóstico participativo, volcamos nuestro trabajo una vez más hacia el ámbito local, a partir de talleres vecinales organizados por cada grupo territorial. De esta manera, el equipo investigador se dirigió a cada sector para realizar talleres de generación de información vinculada a problemas vecinales y su relación con el acceso

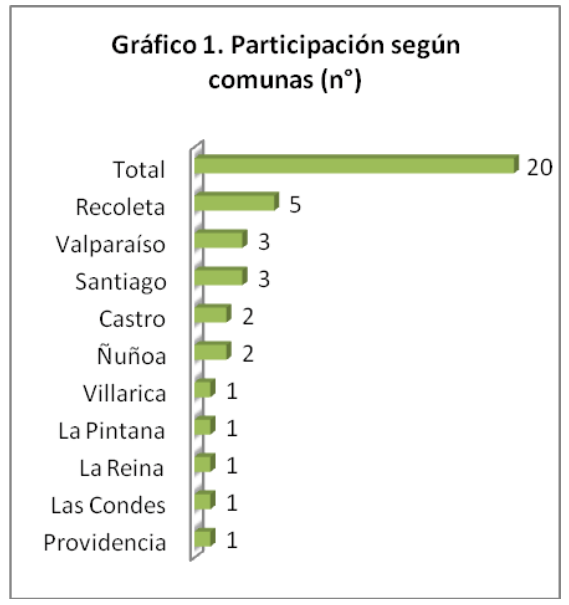
a la información pública. Incluyeron capacitaciones breves, pero sobre todo fueron el instante para salir a recorrer los barrios con los e identificar en terreno las problemáticas que más les afectan. Este trabajo se apoyó en sistemas de información geográfica que permitieron luego generar verdaderos Mapas de la Transparencia Barrial (ver la relevancia de esta herramienta en apartado siguiente). En muchos casos fuimos también más allá del diagnóstico, apoyando también la generación de solicitudes concretas de información al municipio y otros organismos relacionados con los problemas planteados. Estas

\section{Gráfico 2. Participación según tipo de organización (\%)}

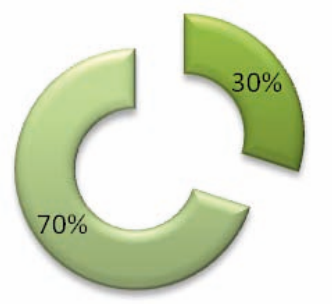

$\square$ Corporación o fundación

$\square$ Organización funcionalo territorial solicitudes fueron apoyadas por el equipo de Ciudad Viva y abogados asesores, como un apoyo concreto a las necesidades barriales, de manera de poder seguir sus respuestas para la investigación.

Cabe señalar por último, que dos de estas solicitudes llegaron a instancias de un reclamo formal ante el Consejo para la Transparencia, con fallos que favorecieron la entrega de información a la ciudadanía (Amparos N C652-10 y C653-10).

Una vez finalizado el proceso, organizamos una jornada de devolución, en que sus resultados fueron presentados a representantes de los barrios participantes y decenas de otros dirigentes y ciudadanos activos, cerrando así un primer ciclo de investigación-acción. 
Cuadro I.Técnicas utilizadas para generación de información.

\begin{tabular}{|c|c|}
\hline Técnica utilizada & Elementos analizados/personas participantes \\
\hline Revisión de antecedentes & $\begin{array}{l}\text { Ley de Transparencia y Acceso a la Información } \\
\text { Pública, Ley General de Urbanismo y } \\
\text { Construcción, Ley de Alcoholes, Planes } \\
\text { Reguladores Municipales, Ley de Juntas de } \\
\text { Vecinos, Programa de Reconstrucción Nacional } \\
\text { en Vivienda. }\end{array}$ \\
\hline Entrevistas a actores clave & $\begin{array}{l}3 \text { vecinos Barrio Bellavista Recoleta } \\
5 \text { vecinos Barrio Bellavista Providencia } \\
2 \text { vecinos Villa Olímpica Ñuñoa } \\
\text { I vecino Cerro Yungay Valparaíso } \\
2 \text { representantes de la Sociedad Civil: Fundación } \\
\text { Pro Acceso y CIPER Chile. }\end{array}$ \\
\hline $\begin{array}{l}\text { Talleres grupales / } \\
\text { marchas exploratorias }\end{array}$ & $\begin{array}{l}44 \text { vecinos / dirigentes en } 4 \text { barrios: } \\
\text { Barrio Bellavista - Recoleta } \\
\text { Barrio Yungay - Santiago } \\
\text { Villa Olímpica - Nuñoa } \\
\text { Cerro Yungay - Valparaíso }\end{array}$ \\
\hline $\begin{array}{l}\text { Taller de capacitación en Ley de } \\
\text { Transparencia dictado por Pro } \\
\text { Acceso. }\end{array}$ & 19 personas \\
\hline $\begin{array}{l}\text { Solicitudes de información - } \\
\text { Ley de Transparencia }\end{array}$ & $\begin{array}{l}46 \text { solicitudes, de las cuales } 30 \text { corresponden a } \\
\text { municipios. }\end{array}$ \\
\hline Comisión y plenario & $\begin{array}{l}20 \text { personas en la Comisión de Transparencia } \\
29 \text { personas en el plenario. }\end{array}$ \\
\hline
\end{tabular}

\section{Antecedentes del acceso a información como Derecho Humano}

En relación con la normativa que ordena el proceso desarrollado, en este apartado revisamos los antecedentes principales de la Ley de Transparencia y una evaluación de su aplicación en nuestro país. Este análisis se sustenta tanto en la revisión bibliográfica como en la aplicación de entrevistas a informantes-actores claves.

En la actualidad muchos países poseen leyes de acceso a la información o mecanismos para ejercer la libertad de información y expresión. De esta manera, se ha instalado como un elemento preponderante en el quehacer de organismos internacionales como la Organización de Estados Americanos, entre otros, que buscan a través de las Relatorías Especiales aportar a los Estados para que garanticen y amplíen el acceso a la información, entendiendo que esta constituye un aspecto fundamental en la consolidación de la democracia y el respeto por los derechos humanos (ver OEA, 2007). 
El derecho de acceso a la información se constituye como un derecho fundamental protegido por la Convención Americana y últimamente ha recibido especial atención por los Estados miembros de la OEA y la jurisprudencia internacional (OEA, 2009).

A pesar de este contexto latinoamericano de creciente interés por mejorar la transparencia en la administración del Estado, antes de la entrada en vigencia de la Ley 20.285 , en nuestro país, tanto los ciudadanos como organismos de la sociedad civil, debían recurrir a otras formas para acceder a la información, y que no necesariamente se respondía en un plazo oportuno o con una respuesta satisfactoria.

(...) nosotros alcanzamos a trabajar casi dos años sin Ley de Transparencia vigente, entonces...cuando la Ley de Transparencia no regía, nosotros quedábamos sujetos a la buena voluntad de la función pública (...) y por tanto todos los documentos, las informaciones que el funcionario público mantenía en su recaudo no fueron conocidos por la ciudadanía. No existía sanción, ni responsabilidad cuando esa documentación se negaba, ni tampoco establecía un procedimiento estándar en el sentido que haya una cierta cantidad de días para que te respondieran (...) (Pedro Ramírez, Centro de Investigación e Información Periodística, CIPER. Entrevista realizada el 10 de agosto de 2010).

En definitiva, se aprecia que la entrada en vigencia de dicha ley confirma una mirada distinta en nuestro país en torno a la transparencia del servicio público y de la administración del Estado. Pese al nivel incipiente de su aplicación, los mismos ciudadanos que la utilizan señalan un cambio en el tipo de respuesta a contar de abril del 2009:

(...) cuando entró en vigencia la ley (...) en general yo diría que $90 \%$ se responden dentro del plazo. Hemos ingresado al sistema solicitudes con 200 peticiones y diría que hay un cambio (...) efectivamente la norma establece una responsabilidad (Pedro Ramírez, CIPER).

En efecto, la transparencia de la administración pública es un gran paso para poner en práctica los mecanismos básicos de la democracia moderna como la participación ciudadana, transparencia, rendición de cuentas y control social de las autoridades, además de colaborar en la disminución de las arbitrariedades y la corrupción.

[La ley constituye] el ejercicio más democrático que hemos tenido en Chile. Tenemos la posibilidad de saber por qué se tomó una decisión en la oficina del alcalde, y cómo se tomó y en virtud de qué se tomó (...) para los ciudadanos es una herramienta muy potente, que es perfectible y mejorable, pero ya existe. Cualquier junta de vecinos, cualquier agrupación barrial o grupo cultural de un barrio puede pedir actuaciones, documentos de su alcalde, de su diputado (Pedro Ramírez, CIPER). 
Por otra parte, el Informe Anual de la Comisión Interamericana de Derechos Humanos, afirma que en una sociedad los derechos políticos requieren de un debate amplio que incluye contar con información pública. En este debate resulta fundamental el papel del Estado, el cual tiene la obligación de promover una cultura de transparencia, divulgar el derecho y las formas de acceso a información (OEA 2009).

En consecuencia, en nuestro país se ha instalado una agenda política en torno a la transparencia de la gestión pública, desde principios de los años 90 , respondiendo entre otras cosas a los problemas suscitados por actos de corrupción y de acuerdo a las recomendaciones internacionales (Rehren, 2008).

En este contexto, la Ley 20.285 entra en vigencia el 20 de abril de 2009 con el fin de regular la publicidad de la información en torno a la administración del Estado. De acuerdo a este principio de transparencia de la función pública, se clarifica el hecho de que cualquier persona puede solicitar información, sin discriminación ni necesidad de señalar los motivos de la solicitud. Además, instala mecanismos para el aprendizaje, utilización e instalación de esta herramienta entre los propios funcionarios de la administración del Estado, en que todo organismo debe proporcionar información "en los términos más amplios posibles, excluyendo sólo aquello que esté sujeto a las excepciones constitucionales o legales” (Alianza Regional por la Libertad de Expresión e Información, 2009, p. 19).

En definitiva, se aprecia un interés desde el Gobierno de Chile por instalar legalmente los mecanismos que permitan crear una cultura de transparencia en la sociedad chilena, tanto desde el quehacer de la administración pública como desde el derecho de todo ciudadano y ciudadana a mantenerse informado sobre la acción de sus gobernantes. De tal manera, nos situamos en un escenario de creciente incorporación de la transparencia como un tema fundamental en el sistema democrático existente. Dentro de las potencialidades que observamos en la profundización de este ámbito (Chile Transparente, 2008), encontramos:

- Reduce la desigualdad en acceso a información de toda la ciudadanía.

- Fortalece empoderamiento ciudadano sobre la gestión de sus gobernantes.

- Fortalece la democracia.

- $\quad$ Está orientada al control ciudadano.

De ahí la importancia y la creciente necesidad de instalar la transparencia y acceso a la información pública como un derecho en el imaginario social del país, como un aspecto que contribuye a fortalecer los canales de participación y democratización existentes, además de fomentar la lucha contra la corrupción. La utilización de esta ley por los ciudadanos puede contribuir a generar aprendizajes e incidencia por parte de las comunidades, a través de una normativa que tiene directa relación con sus prácticas y problemas cotidianos.

Si esa persona se enfrenta en su vida a un problema en su barrio, de seguridad ciudadana, de transporte público, de instalación de una industria contaminante, 
lo que sea, y descubre que existe esta norma y que le sirve (...) y la va a ocupar para todo después (...) puedo saber cómo se eligió el director del colegio de mi hijo por ejemplo. Entonces cuando internalizas una norma como una cosa que afecta a tu calidad de vida o que tiene efectos sobre tu vida cotidiana, dices que eso te sirve y te involucras con eso (Pedro Ramírez, CIPER).

\section{Evaluación de la ley a un año de su entrada en vigencia}

A un año de la entrada en vigencia de la ley, el Consejo para la Transparencia ha realizado varias evaluaciones, tanto para ver el nivel de cumplimiento de las obligaciones de transparencia activa ${ }^{3}$ de parte de las instituciones públicas, como acerca del conocimiento de los ciudadanos en torno a la Ley. Lamentablemente, los resultados arrojan un deficiente posicionamiento de la normativa en la población en general, puesto que a junio del 2010 solamente el $20 \%$ de los chilenos conocía la existencia de una ley que permite conocer información de la gestión pública, mientras que solo el 18,7\% declaró haber escuchado hablar del Consejo para la Transparencia (Dazarola, s/f).

Mientras tanto, si analizamos el nivel de utilización de la ley a partir de las estadísticas elaboradas por la Comisión de Probidad y Transparencia (2010), vemos que se realizaron 47 mil 730 requerimientos entre el 20 de abril y el I de septiembre de 2010 . Predominantemente, las solicitudes son ingresadas a ministerios del área social con el 45,2\%, seguidos por los ministerios económicos con el 33,51\% y, por último, por los ministerios políticos con $21,29 \%{ }^{4}$

En el mismo informe, se señala que casi la mitad de las solicitudes $(47,82 \%)$ fueron realizadas desde la Región Metropolitana, el 46,74\% pertenece a personas entre 30 y 49 años de edad y el 64,01\% fue realizado por varones frente al 35,99\% hecha por mujeres. Asimismo, casi la mitad corresponde a solicitudes de información de personas con alta escolaridad, con estudios universitarios (el 47,41\%). Todo esto refleja el grado en que el uso de la ley discrimina a amplios sectores de la población de nuestro país, relevando de esta forma la importancia que tiene la creación de estrategias provenientes de la propia ciudadanía para aumentar cualitativa y cuantitativamente el uso de la ley de forma equitativa.

En este sentido, es importante conocer no sólo la existencia, sino también tener nociones de cómo funciona el aparato público, con sus matices, complejidades y resquicios. Esta es la primera barrera que la ciudadanía debe enfrentar, antes, inclu- 
so, de decidirse a hacer una solicitud de información, a lo que se suman las distintas etapas estipuladas en la propia la ley.

Porque te entregan parte de la información o información que no es la que estás pidiendo o que te la niegan. Son tres tipos de repuesta distintas, que no cumplen con la expectativa que estás buscando. De ahí la urgencia de capacitación para la ciudadanía, ya que la gente sabe que puede pedir cosas en virtud de la ley, pero no saben en cuánto tiempo responden, si pueden apelar, que pueden ir al Consejo para la Transparencia y a la Corte de Apelaciones si el Consejo no es satisfactorio (Pedro Ramírez, CIPER).

Por otro lado, un estudio del Centro de Derechos Humanos (2009) muestra que en torno al primer año de entrada en vigencia de la Ley, existen múltiples deficiencias provenientes en su mayoría del funcionamiento o aplicación de la misma en el sector público. Algunas falencias detectadas son las siguientes: ${ }^{5}$

I. El plazo destinado para su implementación es mucho menor en comparación con otros países, lo que va en desmedro de una real interiorización de los funcionarios y la ciudadanía de esta herramienta. Esto genera su aplicación sobre el terreno de la improvisación y deficiencias recurrentes.

2. Escasa difusión o pedagogía de la ley hacia la ciudadanía, lo que imposibilita un real control ciudadano.

3. Desigual capacitación para funcionarios entre los distintos servicios públicos, asimismo un mínimo porcentaje del total de estos poseen capacitación.

4. Inconvenientes ocasionados por la gran variabilidad que presenta la transparencia activa entre los distintos organismos. La información no se presenta con facilidad ni integralidad, lo que manifiesta la necesidad de crear un portal único de Transparencia Activa.

Esta variabilidad en la aplicación de la ley, se aprecia con mayor intensidad a nivel municipal, aspecto que veremos más adelante a raíz de esta investigación participativa, respecto a las graves falencias existentes en el funcionamiento de la normativa en los gobiernos locales.

(...) creo que hay un problema porque en general las entidades del estado como ministerios, intendencias tienen un sistema unificado de gestión de solicitudes. Eso no ocurre con los municipios (...) entonces te encuentras con distintos tipos de formularios por ciertos municipios (...). Hay que unificar los criterios y hacer un formulario único (Pedro Ramírez, CIPER). 
Más adelante observaremos algunas deficiencias detectadas en el ámbito municipal, al utilizar las herramientas que otorga la Ley de Transparencia en casos concretos de distintas organizaciones ciudadanas, especialmente a nivel barrial.

\section{Diagnóstico participativo-sistematización de solicitudes}

Con el objetivo de generar información territorial referente a las principales áreas de déficit en transparencia a nivel municipal realizamos entrevistas a vecinos y talleres vecinales. En ellas utilizamos las técnicas del mapeo y las solicitudes de acceso a la información pública para el diagnóstico y la generación de información. Con ambas herramientas, buscamos desarrollar un aprendizaje conjunto sobre la Ley de Transparencia, además de la reflexión y el compromiso de los vecinos sobre temas específicos que requieren de mayores antecedentes para la gestión barrial.

Utilizamos los sistemas de información geográfica (SIG) como un instrumento innovador y muy potente para la gestión barrial ciudadana, que aporta a la transparencia en la relación barrio-municipio-autoridades. El mapeo permite "espacializar" situaciones o eventos que ocurren dentro de los territorios y encontrar patrones que se repiten y que inciden en el tipo de barrio sector que estamos construyendo. También permite visibilizar los conflictos de uso al interior de los territorios, lo que contribuye a ampliar los niveles de transparencia hacia los ciudadanos. En definitiva, realizamos cuatro talleres, entre octubre y diciembre 2010 , con el objetivo de potenciar y dar mayor validez técnica a los diagnósticos que los vecinos hicieron de sus barrios, contribuyendo a generar un diálogo en igualdad de condiciones entre la ciudadanía y los organismos públicos.

Paralelamente, entre junio y noviembre de 2010 realizamos un total de 30 solicitudes de información tanto desde CiudadViva como de organizaciones barriales y vecinos de los cuatro barrios involucrados. Adicionalmente, (julio 20I0) hicimos 16 solicitudes en el contexto de la capacitación dictada por la Fundación Pro Acceso. En total, 46 solicitudes son objeto de esta mirada exploratoria.

Las solicitudes se realizaron sobre la base de una serie de problemáticas identificadas en el diagnóstico desde las propias

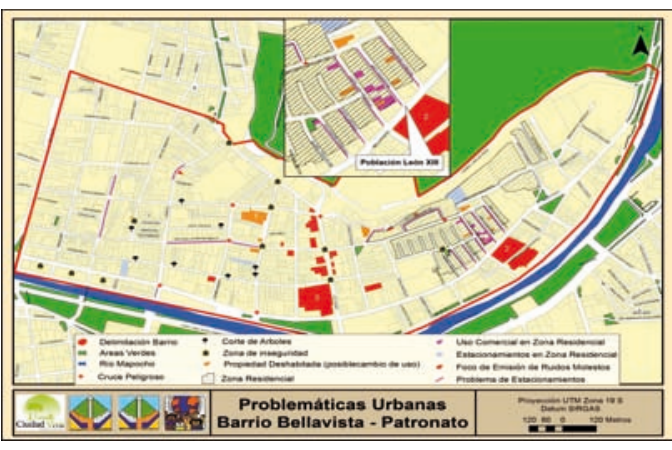

Mapa I. Bellavista-Patronato comunidades. Estos problemas se sustentan en las propias experiencias de los vecinos residentes en sus barrios, con frecuentes falencias en la gestión del territorio que requieren de la intervención activa de la propia ciudadanía. El equipo de CiudadViva apoyó en el seguimiento de los procesos de solicitud de información en el marco de la Ley de 
Transparencia. Además de estas solicitudes emanadas de los vecinos, desde Ciudad Viva realizamos directamente otros requerimientos, con el fin de georreferenciar situaciones relacionadas con déficit de transparencia a nivel barrial o territorial, sobre todo en el Barrio Bellavista, sector en el que estamos emplazados como organización de base. Por lo tanto, el origen de ciertas solicitudes varía de acuerdo a las necesidades de información y al objetivo de su realización.

$\mathrm{Si}$ analizamos los temas a que aluden las solicitudes realizadas, podemos ver en el gráfico 3 que los temas más consultados tienen relación con las patentes de alcohol, los usos de suelo y los permisos de demolición, confirmando que la conformación urbana de cada barrio es de vital importancia para los vecinos. Mención aparte tienen las solicitudes relacionadas con la emergencia y reconstrucción posterremoto 2010. Además, se pregunta por temas vinculados a la gestión patrimonial, la recuperación de espacios públicos $y$, en menor medida, sobre materias asocia-

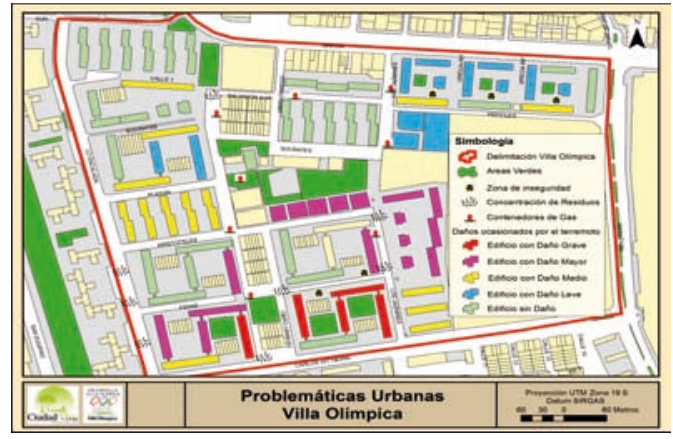

Mapa 2.Villa Olimpica das a las juntas de vecinos y reciclaje. ${ }^{6}$ De acuerdo a esta constatación trabajamos con representantes de las distintas comunidades en propuestas concretas para mejorar los niveles de transparencia relacionados con estos temas, que presentaremos más adelante.

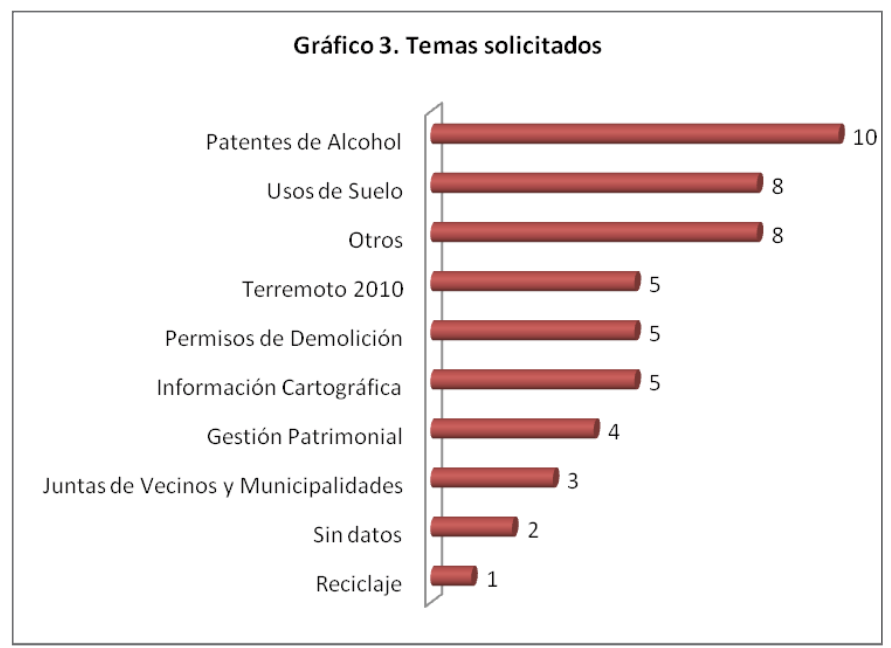

6 Los casos señalados como Otros corresponden a solicitudes variadas como alza de contribuciones, gastos en obras de construcción, condiciones laborales en empresas particulares, seguridad pública, partes cursados a una propiedad, por ejemplo. Todas solicitudes ingresadas para diferentes comunas. 
Por otra parte, el gráfico 4 muestra los organismos a los cuales se dirigieron las solicitudes de información.Aquí los municipios obtienen mayor proporción (65\%), demostrando su preponderancia en los temas de gestión barrial. En segundo lugar, en los ministerios recae el $13 \%$ de los requerimientos, seguidos en tercer lugar con el $5 \%$ Carabineros de Chile y el Consejo de Monumentos Nacionales en aquellas situaciones que requieren de gestión patrimonial. Por último, con el $2 \%$ de las solicitudes tenemos a organismos como la Armada de Chile, la Contraloría General de la República y el Consejo para la Transparencia, entre otros.

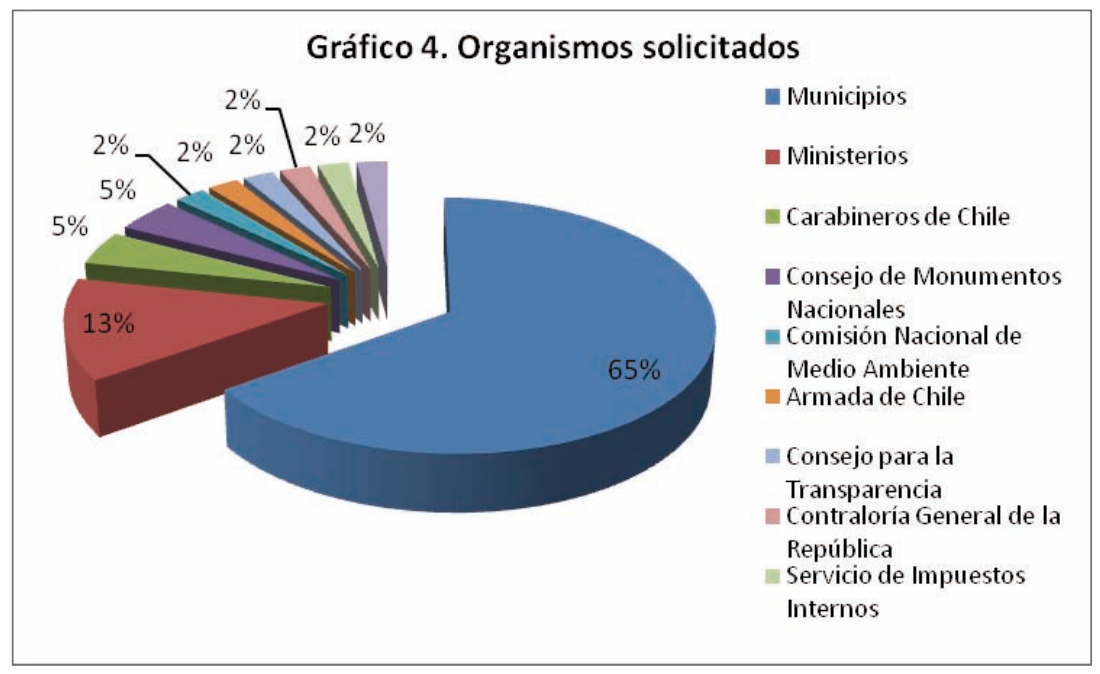

Por último, para una mirada de las respuestas obtenidas en las distintas solicitudes, observamos el cuadro siguiente que evidencia en forma sintética, la variedad de respuestas que emanan de los organismos públicos a pesar de la vigencia de la normativa.

Cuadro 2.Tipos de respuestas obtenidas en las solicitudes.

\begin{tabular}{|l|c|}
\hline \multicolumn{1}{|c|}{ Tipos de respuestas } & $\mathrm{N}^{\circ}$ \\
\hline Dentro del plazo con información completa & 20 \\
\hline Dentro del plazo con información parcial & 4 \\
\hline Fuera de plazo con información completa & 4 \\
\hline Dentro del plazo con denegación & 3 \\
\hline Dentro del plazo retirar información en organismo & 2 \\
\hline Esperando respuesta & 2 \\
\hline Dentro del plazo con denegación parcial & $\mathrm{I}$ \\
\hline Dentro del plazo con derivación pero sin respuesta & $\mathrm{I}$ \\
\hline Dentro del plazo, solicitan aclarar solicitud & $\mathrm{I}$ \\
\hline
\end{tabular}




\begin{tabular}{|l|c|}
\hline $\begin{array}{l}\text { Fuera de plazo retirar información en organismo, } \\
\text { cancelando valor }\end{array}$ & $\mathrm{I}$ \\
\hline Uso de prórroga y respuesta completa & $\mathrm{I}$ \\
\hline Uso de prórroga y sin respuesta & $\mathrm{I}$ \\
\hline Sin datos I & 5 \\
\hline Total & $\mathbf{4 6}$ \\
\hline
\end{tabular}

En este sentido, vemos que en la mayoría de los casos se respetan los plazos dados por la ley, pero las respuestas pueden entregar información parcial o derechamente denegarla. Para esto último, por lo general, se alude al artículo 2 I, $\mathrm{N}^{\circ} \mathrm{I}$ letra c de la ley, que indica que la entrega de información puede distraer a los funcionarios de sus labores habituales.Asimismo, algunas entidades utilizan el derecho de prórroga de 10 días, pero no siempre terminan entregando lo solicitado.

A pesar de que en la solicitud se indica el medio en que debe ser entregada la información (correo postal, correo electrónico), algunos funcionarios solicitan el retiro de la documentación en el mismo organismo, previa cancelación de un valor, que algunas veces escapa a lo permitido por la ley (por ejemplo, $\$ 40$ por hoja fotocopiada, $\$ 37.000$ por un CD).

\section{Hallazgos de la aplicación municipal de la Ley 20.285}

A partir de esta información y de acuerdo a la experiencia de aplicación práctica y monitoreo de solicitudes ciudadanas, identificamos aspectos de gran importancia para la utilización de esta herramienta por las comunidades. También encontramos una serie de inconvenientes y barreras para hacer correcto uso de la Ley de Transparencia. A continuación revisaremos algunos hallazgos generales que nacen de la experiencia directa de utilizar la ley por parte de la ciudadanía y sus agrupaciones, y que constituyen obstáculos en el acceso a información pública:

- En algunos organismos existe total desconocimiento de la Ley de Acceso a la Información Pública y del procedimiento a seguir para entregar información, lo que demuestra graves falencias en los procesos de capacitación de los funcionarios públicos que las autoridades realizaron al entrar en vigencia la ley. Este aspecto se complementa con una actitud de estos que no facilita el proceso de entrega de información. Lo anterior es especialmente pertinente para el ámbito municipal.

- En algunos organismos solamente se cumple con Transparencia Activa, con total ausencia de la opción de Transparencia Pasiva. Por ejemplo en municipios como Recoleta y Valparaíso no existe opción alguna para solicitar información específica, ya sea por formulario electrónico o una dirección determinada para hacer entrega del impreso. 
- $\quad$ Falta uniformidad en la manera de presentar la sección de transparencia en cada municipio. Esto ocurre tanto en Transparencia Activa como Pasiva, lo que repercute en la accesibilidad a las páginas y, por supuesto, a la información requerida.

- La Transparencia Activa se presenta de forma compleja, poco comprensible y poco expedita para la comunidad, especialmente para aquellos sin un conocimiento especializado en estas materias. Esto es complicado si entendemos que el derecho de acceder a la información pública debe ser un proceso más asequible para las personas, sin importar su nivel educativo.

- Escasa difusión hacia la ciudadanía. Aún se percibe poco de algunos municipios para que vecinos y vecinas conozcan y usen este derecho. Otros organismos tampoco se han hecho cargo en su mérito de la falta de información al respecto.

- Problemas en el envío electrónico de la solicitud, por ejemplo esto ocurre con el municipio de Ñuñoa o Pedro Aguirre Cerda, donde a pesar de presentar la opción por formato electrónico, este se torna dificultoso y se debe insistir varias veces hasta cumplir con el procedimiento. Es decir, se entrega la herramienta, pero esta no funciona cabalmente.

- Algunos organismos piden datos personales para hacer la solicitud, por ejemplo haciendo obligatorio crear un usuario web, ingresando Rut o domicilio para lograr acceder a información, aspecto que en la ley no está contemplado.

Cabe recalcar que los anteriores, son problemas generales identificados directamente por los dirigentes y vecinos de los barrios que participaron del proceso, así como por el equipo profesional que monitoreó y documentó la experiencia. Si bien los resultados no tienen una representatividad estadística, sí corresponden a casos muy representativos del quehacer ciudadano, en tanto resultan de casos concretos de organizaciones ciudadanas consolidadas o pioneras, realizados en el marco de su trabajo cotidiano en la gestión de sus barrios. En ese sentido su representatividad cualitativa es importante y amerita la realización de nuevos estudios y de mayor magnitud para corroborar los hallazgos encontrados.

\section{Propuestas}

De acuerdo a los distintos temas de interés ciudadano que pudimos identificar en los diagnósticos participativos, así como las barreras y los problemas que surgieron de las solicitudes de acceso a la información pública realizada por los vecinos y vecinas, presentamos una serie de propuestas que integran la participación ciudadana en distintos ámbitos de la transparencia y acceso a la información pública. Nos enfocamos especialmente en el nivel municipal, por ser la primera línea del Estado frente a las personas, así como por su pertinencia en la solución de los problemas barriales. Estas propuestas serán las que configuren el área de transparencia en la gestión urbana en la Agenda Ciudadana.

En primer lugar, presentamos propuestas generales sobre distintos componentes que intervienen en la aplicación de la ley. En segundo lugar, exponemos 
propuestas relacionadas específicamente con el trabajo de las organizaciones y los ciudadanos activos dedicados a la gestión territorial de sus barrios, en especial de Bellavista, Yungay y Villa Olímpica en Santiago, así como Cerro Yungay en Valparaíso. Luego, exponemos un cuadro que sintetiza cada una de las propuestas con metas e indicadores para su seguimiento. Por último, presentamos algunas propuestas relacionadas con la transparencia a nivel de la sociedad civil y algunos estudios adicionales que creemos necesarios para apoyar este proceso de profundización democrática.

\section{I Propuestas generales}

\section{I.I Difusión del derecho al acceso}

En primer lugar, dado el desconocimiento de la ley que pudimos comprobar, tanto en funcionarios públicos como en otros sectores de la población, y con el fin de construir una mayor participación ciudadana y profundización democrática, planteamos que es necesario ampliar el enfoque y las acciones que se realizan en torno a la difusión de la ley, especialmente para instalarla desde una perspectiva de derecho a la información pública. Por eso planteamos:

- Que el Estado incorpore efectivamente mecanismos de facilitación para el aprendizaje de la ley por la ciudadanía, para evitar su uso siga replicando las inequidades típicas del país (hombres, alta educación, residentes en Santiago, etc.). Esto implica diversificar las fórmulas de capacitación para distintos segmentos sociales.

- Implementar procesos de difusión y capacitación de la ley en colegios municipales de cada comuna y elaborar campañas de difusión en medios de comunicación masivos y locales. Esto se puede realizar a través de una coordinación directa con el Consejo para la Transparencia y otras instituciones.

- Estandarizar el nivel de conocimiento de la ley entre los funcionarios públicos. Para esto se requiere instalar estándares de capacitación aplicados en forma sistemática. Por ejemplo, en algunos países se han adoptado guías para funcionarios (OEA, op. cit) que intentan nivelar los procesos de aprendizaje en torno a la transparencia y el acceso a información pública.

El problema de la educación se ha enfrentado de manera importante en otros países. La ley nicaragüense, por ejemplo, prevé tanto el entrenamiento de funcionarios como la incorporación del derecho a la información en los currículos escolares a todos los niveles. Incluso considera el establecimiento de un centro nacional de investigación y docencia sobre el derecho a la información. $O$ también Corea del Sur, en donde el Sistema OPEN es por una campaña de información en todo Seúl (con adhesivos y folletos) y en los medios de comunicación, además de la elaboración de guías para la utilización del sistema. 


\section{I.2. Mejoramiento del acceso y funcionamiento de la ley}

Como ya hemos revisado, la utilización de la ley por parte de la ciudadanía se enfrenta a variados inconvenientes de forma y contenido, especialmente a nivel municipal, ya sea por falta de acompañamiento, recursos o tiempo, o simplemente por dejación. Esto se traduce en una debilidad institucional respecto al funcionamiento de la Ley.

Más allá de los esfuerzos hechos por estudios como los realizados por el Instituto Chileno de Estudios Municipales (2009-2010) y esta misma investigación participativa, buscando iluminar lo que sucede en el ámbito local, hoy día no sabemos con certeza qué está pasando a nivel municipal con el acceso a la información pública, pues aún faltan estudios con la profundidad necesaria. Esto se explica en parte por la diversidad existente a nivel municipal, en términos de recursos, tamaño, etc., pero también por un cierto abandono desde el nivel central. De esta forma, no existe un criterio estandarizado para entregar la información por parte de las municipalidades, ni se les fiscaliza efectivamente para que lo tengan. Esto genera una peligrosa variedad en la gestión de la información y posterior entrega a los ciudadanos. De ahí la necesidad de crear mecanismos de monitoreo sistemático en torno a cómo está siendo utilizada o no la ley en el nivel local, con un fuerte componente de participación en la evaluación y seguimiento tanto de la población en general, como de organizaciones de la sociedad civil e instituciones académicas. Por ello proponemos:

- $\quad$ Realizar estudios de mayor profundidad a nivel municipal, que permitan conocer mejor lo que está sucediendo en ese ámbito. El trabajo de la Universidad Autónoma (ICHEM) en este sentido ha sido importante, pero se necesita aún más. Por ejemplo a nivel de brechas técnicas, de expectativas, barreras estructurales, intracomunales, etc.

- Crear un portal único de acceso a la información pública, que permita acceder desde un solo lugar a las distintas reparticiones del Estado, pero que además oriente al usuario, señalando los distintos pasos que implica hacer solicitud (prórrogas, quejas, apelaciones, etc.), que haga públicas las respuestas obtenidas (para evitar duplicaciones y trabajo extra), estadísticas acabadas del proceso, etc. Creemos que es necesario instalar mecanismos serios que posibiliten la accesibilidad y uso creciente de la ciudadanía tanto de Transparencia Activa como Pasiva. En Seúl, Corea del Sur, se utilizan las nuevas tecnologías para la transparencia y la lucha contra la corrupción (Sistema OPEN), un sitio web que permite a los ciudadanos solicitar información pública administrativa, o pedir permisos (de construcción por ejemplo) y también se pueden encontrar informaciones sobre el proceso de entrega del documento pedido.

- Construir estándares e indicadores de transparencia, para evaluar el desempeño de la gestión pública, especialmente municipal. Bastantes países consideran un estándar mínimo para el manejo de registros.Algunos de ellos, como México, le ceden el poder a un órgano central, el Instituto Federal de Acceso a la Información, de establecer estándares para el manejo de registros, además de un sistema 
para asegurar que las entidades públicas respeten estos estándares. Éste es un buen enfoque, ya que puede asegurar estándares fuertes y uniformes para todo el sector público. Por otro lado, en Corea del Sur, por ejemplo, se monitorea si hay retraso en la publicación de la información, con premios para los mejores y sanciones para los que tienen retraso o que no dan buena información.

- Generar líneas de base e indicadores de los avances obtenidos en los temas de mayor preocupación ciudadana, para monitoreo de la gestión pública. Estos indicadores deben ser de conocimiento público y con participación de la ciudadanía. De esta forma se realiza un seguimiento y control con mayor incidencia de los mismos vecinos en la gestión de sus barrios. Esto apoya un cambio cultural para el posicionamiento del acceso a la información como un derecho humano. Para el ámbito territorial, proponemos además incorporar crecientemente el uso de los SIG como herramienta útil para la gestión urbana como para mejorar el uso y aplicación de la ley, identificando zonas de mayor o menor transparencia, localización de problemas específicos de déficit de transparencia, etc.

\section{I.3. Fortalecer la institucionalidad democrática referente al acceso}

Dado que el Consejo para la Transparencia posee un rol fundamental en el funcionamiento de la ley y en la disminución de asimetrías en su utilización por parte de la ciudadanía, creemos necesario fortalecer las facultades de este organismo, pero acompañado de una fuerte presencia de la ciudadanía en su conformación. Para esto proponemos:

- Generar mecanismos de elección pública de los consejeros, sino de todos, por lo menos de una parte de ellos. Hoy día esta facultad reside directamente en el Presidente de la República, con ratificación del Senado, pero al tratarse de una ley referida a la regulación del Estado y el acceso desde la sociedad civil, es necesario mejorar el accountability de esta entidad hacia la ciudadanía y asegurar más su independencia frente al Gobierno de turno. Además, esto implicaría mayor conocimiento e información de la ciudadanía frente al ente que fiscaliza y garantiza el derecho. Formas de elección se aplican ya en entidades como el Instituto de Derechos Humanos o la Corporación Nacional de Desarrollo Indígena, por lo que existe una experiencia que puede ser replicada.

- Presupuesto autónomo y plurianual para el Consejo, para evitar que año a año este organismo deba pelear por sus recursos, con el riesgo de que sean cada vez menos. Esto primero para permitir una mejora planificación a largo plazo del organismo (incorporando mejor, por ejemplo, sus labores educativas), pero por sobre todo porque no corresponde que el financiamiento de esta instancia dependa año a año de quienes precisamente son fiscalizados por él. 


\subsection{Propuestas temáticas}

Como ya hemos mencionado, la Ley de Transparencia es una herramienta excepcional para mejorar la gestión barrial, pero no debemos olvidar que el foco debe estar puesto en los problemas que efectivamente enfrentan vecinos y vecinas en sus territorios (en el fin, no en el medio). El problema de la transparencia y la eficiencia en la gestión pasa, por un lado, por el acceso a la información pública, pero por sobre todo, implica hacer partícipe a la comunidad en las políticas implementadas en su ciudad. De esta manera, la ciudadanía puede ejercer realmente sus atribuciones de fiscalización y control, pero además se hace parte de la solución.

Por eso planteamos acá algunas medidas que hemos identificado a partir del trabajo de los grupos involucrados en esta investigación, que mejorarían de manera notable la gestión territorial urbana mediante la información y participación. Estos aportes provienen básicamente del ámbito local, en el cual establecimos nuestro trabajo. De todas formas, hacemos una propuesta de Transparencia Activa Municipal, que se refiere a información básica que la mayoría de las organizaciones territoriales necesitan y utilizan día a día, y que debería estar publicada permanente por parte de los municipios.

Reiteramos que todas estas propuestas fueron debatidas y enriquecidas en diversas oportunidades con vecinos y representantes de organizaciones de la sociedad civil, especialmente aquellas con arraigo territorial.

\subsection{Planos reguladores}

Entre los intereses de los grupos ciudadanos urbanos, destaca la importancia de los planos reguladores en el diseño urbano y su relación con mejorar la calidad de vida de las comunidades. Por esto, recomendamos:

- Crear mecanismos de difusión o mejorar los existentes en torno a este instrumento de planificación territorial. Resulta necesario dar a conocer en qué consiste y la importancia que tiene el plan regulador en la gestión de la ciudad y en la gestión local, toda vez que al adquirir familiarización con este instrumento, la ciudadanía posee una capacidad potente de ejercer control de los usos y destinos para cada lugar del barrio, generar propuestas en sitios eriazos, fiscalizar usos ilegales, entre otros.

\subsubsection{Ley de alcoholes}

El problema de las patentes de alcohol se presenta de forma diferenciada en cada comuna, sin embargo siempre representan un foco de nubosidad en torno a los procedimientos que realizan los gobiernos locales para fiscalizar el cumplimiento correcto de la normativa. Este problema se vive intensamente en Bellavista y sectores de Valparaíso. Además de las propuestas sobre transparencia activa, que enumerare- 
mos más adelante, creemos que se puede hacer un trabajo fructífero sobre la base de sistemas de información geográfica, por eso proponemos:

- Que los municipios elaboren mapas de la concentración territorial de patentes de alcohol de los distintos tipos. Existen especificaciones en los usos para patentes de alcohol que varían en la misma ley y en la permisividad que otorga el Plano Regulador.A su vez, hemos visto, especialmente en el Barrio Bellavista, que su concentración tiene directa relación con situaciones de violencia y delincuencia, por lo que trabajar con este tipo de información resulta un potente insumo para la gestión local entre Gobierno y ciudadanía en materias de seguridad pública.

Por otra parte, dada la importancia que adquieren los grupos ciudadanos al asumir un rol fiscalizador y de mayor protagonismo en la gestión de sus barrios , proponemos el uso de la Ley 20.285 por las comunidades a través de Transparencia Pasiva para que puedan controlar:

- El detalle de las peticiones de patentes de alcohol ingresadas al municipio y cuáles de ellas han sido aprobadas en un período determinado.

- Las fiscalizaciones municipales realizadas a domicilios con patentes de alcohol y sus resoluciones.

- La gestión municipal en el control de las externalidades que generan estos usos.

Esto último apunta a las propias organizaciones ciudadanas, mejorando su incidencia a nivel local. Es decir, a través de la transparencia promovemos y activamos mayor participación e incidencia de las comunidades en su territorio.

\subsubsection{Seguridad pública}

Las posibilidades que posee la ciudadanía actualmente para conocer y participar de los planes de seguridad de su sector son reducidas, toda vez que la orientación que han adquirido las políticas públicas en este tema es de deficiente involucramiento activo de la población.

Como sabemos, la inseguridad que experimentan las comunidades puede ser abordada a través de diversos frentes (prevención individual, comunitaria, situacional, tratamiento y rehabilitación, reinserción social, entre otros). No obstante, rara vez la población accede en forma oportuna a esta información y mucho menos conoce los canales efectivos de participación a nivel local. Un ejemplo claro de esto ocurrió en La Legua. Por esto proponemos:

- Que se generen instancias de coordinación con el gobierno local y con el plan cuadrante correspondiente, previa utilización del derecho de acceso a información, que permita lograr participación y fiscalización en las acciones desarrolladas desde el sector público en esta temática. 
- Que los organismos encargados de la seguridad (municipalidad, Carabineros de Chile, Policía de Investigaciones), utilicen sistemas de información geográfica para presentar de forma didáctica los principales elementos de las políticas locales en seguridad. De esta forma, la ciudadanía puede gestionar la seguridad de su territorio de manera más clara y concreta.

\subsubsection{Gestión patrimonial}

Dado el desconocimiento que existe en torno a las declaraciones patrimoniales de nuestros barrios recomendamos:

- Elaborar y publicar un catastro de las zonas patrimoniales en la comuna, los instructivos respectivos y las implicancias para la comunidad.

Esto es especialmente pertinente para barrios de carácter patrimonial como Bellavista y Yungay.

\subsubsection{Plan de reconstrucción posterremoto}

Posterior al sismo de febrero de 2010, el daño ocurrido en términos de infraestructura, a nivel psicológico, organizativo y de redes, entre otros, ha generado graves y urgentes reestructuraciones en los lugares afectados. En este contexto, la participación de la comunidad resulta fundamental para superar este proceso y aportar en la restauración y (o) reconstrucción de cada territorio. Sin embargo, algunas de las comunidades afectadas por el terremoto que participaron de esta investigación, señalan no contar con la información necesaria sobre el proceso y sus etapas y, peor aún, no se han sentido verdaderamente partícipes de este. Es el caso de la Olímpica. En este sentido, proponemos:

- Que los planes de reconstrucción se encuentren publicados por Transparencia Activa de un organismo público que centralice esta información, por ejemplo el MINVU. Creemos que la comunidad debe tener acceso en detalle a la información relacionada con la reconstrucción de sus sectores. La finalidad es lograr que la comunidad organizada utilice la Ley de Transparencia y los mecanismos complementarios que coadyuven en la real democratización y el fortalecimiento de la ciudadanía en materias de gran impacto como esta.

- Que las políticas públicas de reconstrucción incorporen activamente a la ciudadanía, tanto en el diseño como en la implementación de este proceso. Aspecto que debería ser aplicado a cualquier situación de desastre.

\subsubsection{Propuestas de Transparencia Activa a nivel municipal}

A continuación, el cuadro 3 sintetiza los puntos básicos que proponemos incorporar en la sección de Transparencia Activa a nivel municipal. Se basan en la información 
que más básica y comúnmente necesitan las agrupaciones ciudadanas en la gestión de sus territorios, según la experiencia de los grupos de experiencia con los que trabajamos. Proponemos esto con el fin de economizar el tiempo de los funcionarios municipales y de la ciudadanía, así como también lograr mayor transparencia de la gestión pública.

Cuadro 3. Propuestas de Transparencia Activa según temáticas.

\begin{tabular}{|c|}
\hline al \\
\hline Planes reg \\
\hline $\begin{array}{l}\text { I. Todo permiso de demolición y edificación, indicando propietarios, si existe cambio } \\
\text { de uso, el proponente y donde se puede expresar opinión respecto a los cambios } \\
\text { que esto provoca en el barrio. } \\
\text { 2. Aspectos principales del Plan Regulador Comunal, en qué consiste, su importancia } \\
\text { en la conformación de los barrios, mecanismos de participación. }\end{array}$ \\
\hline Normativa de ruido \\
\hline $\begin{array}{l}\text { I. Aspectos principales de la normativa de ruidos, en qué consiste, mecanismos de } \\
\text { incidencia de los vecinos en fiscalizar ruidos molestos. }\end{array}$ \\
\hline Ley de alcoholes \\
\hline $\begin{array}{l}\text { I. Rol actualizado de patentes de alcohol vigentes en la comuna, por tipo, con detalle } \\
\text { de domicilio y fecha de otorgamiento. } \\
\text { 2. La ordenanza municipal respecto a los horarios de cierre de las patentes de alco- } \\
\text { hol. }\end{array}$ \\
\hline Gestión patrimonial \\
\hline $\begin{array}{l}\text { I. Catastro de monumentos nacionales de todo tipo en la comuna, instructivos res- } \\
\text { pectivos y las implicancias para la comunidad }\end{array}$ \\
\hline Plan de reconstrucción posterremoto \\
\hline $\begin{array}{l}\text { I.Todas las resoluciones adoptadas para los planes de reconstrucción de las viviendas } \\
\text { afectadas, los estudios realizados y análisis que fundamentan la gestión pública en este } \\
\text { tema. } \\
\text { 2. Procedimientos para la obtención de subsidios para las personas afectadas, los } \\
\text { criterios establecidos y el detalle de aquellos subsidios asignados. }\end{array}$ \\
\hline Seguridad pública \\
\hline $\begin{array}{l}\text { I. Dotación policial existente en cada comuna y cuadrante. } \\
\text { 2. Información detallada de las políticas locales ejecutadas para seguridad, proyectos, } \\
\text { planes, así como el rol que cumple cada institución: municipio, Policía de Investigacio- } \\
\text { nes, Carabineros. }\end{array}$ \\
\hline
\end{tabular}

\subsection{Transparencia en la sociedad civil}

La necesidad de transparentar las acciones de cualquier institución, así como la rendición de cuentas ante los ciudadanos corresponde a un principio fundamental para mejorar la calidad de cada organismo y fortalecer los procesos democráticos de un país. 
Si queremos construir una verdadera cultura de la transparencia, las organizaciones de la sociedad civil no sólo deben cumplir un rol fiscalizador y de control del aparato público, sino que ellas mismas deben también predicar con el ejemplo. Es por ello que nuestra agenda ciudadana no sólo exige a la autoridad, sino que también implica autoimponernos compromisos desde la ciudadanía.

En este sentido, proponemos generar estándares y prácticas de transparencia también al interior de las organizaciones ciudadanas, para hacerse verdaderamente cargo del rol que ellas deben cumplir. Los estándares de transparencia para ONG desarrollados por Chile Transparente (2008) son una buena manera de comenzar.

Así también las juntas de vecinos, uniones comunales y otras agrupaciones territoriales deben ser referentes propios dentro de su comunidad a nivel barrial, y pueden levantar temas propios de cada territorio para ser de conocimiento público entre los vecinos, con el fin de favorecer procesos participativos y generadores de protagonismo de la población en general en la gestión de sus barrios.

\subsection{Propuestas de estudios o investigaciones}

A continuación presentamos algunas propuestas para fomentar el estudio y las investigaciones para mejorar nuestro conocimiento y prácticas en torno a la transparencia, que pueden ser abordadas desde el sector tanto público como académico.

- Crear línea de base en transparencia a nivel municipal, como punto de partida para evaluaciones en este ámbito.

- Análisis y (o) sistematización de experiencias y estándares internacionales en transparencia.

- Con la creación del portal único de acceso a información (ver propuestas generales), generar datos duros más acabados de acuerdo a su utilización y desarrollo en el tiempo.

- Análisis cualitativos sobre qué se solicita y cómo se responde, más allá de la información estadística dura.

- $\quad$ Análisis jurídicos sobre los fallos del Consejo para la Transparencia, especialmente en la perspectiva de derechos humanos.

- Incentivar el desarrollo de cursos universitarios y tesis de grado en torno a la transparencia.

- Instaurar un sistema de monitoreo y evaluaciones periódicas de las autoridades políticas, basadas en el uso de la ley, pero en relación a las temáticas de interés ciudadano, con apoyo de la academia. Esto permitiría realmente darle un realce al sistema en base a la profundización democrática y rendición de cuentas hacia la ciudadanía. 


\section{Conclusiones}

El derecho de acceso a la información y el principio de transparencia y la rendición de cuentas constituyen ejes fundamentales en la democratización de la gestión de los territorios, donde intervienen tanto actores del Gobierno como de la ciudadanía.

Las organizaciones de la sociedad civil se caracterizan por situar sus fines en la esfera de lo público y disputarle preponderancia al rol anterior asumido de forma predominante por el Estado. Lo principal es que se mueven por intereses colectivos en el marco de un actuar que busca tanto enriquecer la agenda pública, como también controlar y fiscalizar permanentemente la acción del Estado.

Para fortalecer sus buenas prácticas, se requiere adquirir crecientemente un carácter que dispute sus derechos y espacios dentro de la realidad social, ejercer sus responsabilidades y fortalecer su capacidad de incidencia en políticas públicas, en el imaginario social, así como lograr instalarse a nivel territorial con legitimidad y sustentabilidad.

Como hemos revisado en el presente estudio, aportamos con lineamientos que puedan otorgar contenido a un programa en torno a la necesidad de construir ciudades más justas, democráticas e inclusivas, en las cuales el rol ejercido por la sociedad civil al amparo de lo que faculta la ley de transparencia resulta crucial en el segundo año de su entrada en vigencia y en un momento en que se necesita de forma urgente la difusión para una mayor utilización de esta normativa, pero sobre todo que la ciudadanía aprehenda la relevancia que significa asumir el derecho de acceso a la información como un derecho y que, además, es un piso esencial en dar pasos en el terreno del control ciudadano del Estado y en el fortalecimiento democrático.

En consecuencia, se propone a partir de esta Agenda Ciudadana aportar en las políticas públicas y en la generación de herramientas para que los ciudadanos adquieran la titularidad del derecho de acceso a la información como un componente vital en su participación de los temas públicos.

A pesar de las falencias detectadas en la aplicación de la ley y la limitada utilización por ciertos segmentos sociales, confiamos en que constituye una poderosa herramienta para ser utilizada por las personas y los grupos ciudadanos que se involucran con la gestión democrática de sus territorios.

La serie de propuestas entregadas en este informe pretende dar un puntapié inicial a la construcción de estrategias que permitan concretizar estos temas, vincularlos a compromisos ciudadanos y buscar componentes medibles a mediano y largo plazo para su control permanente. En este sentido, aportamos con un insumo a ser profundizado y actualizado basado en la experiencia compartida con las organizaciones sociales asociadas a estos temas. 


\section{Bibliografía}

Alianza Regional por la Libertad de Expresión e Información. (2009). Saber Más. Informe Regional sobre la situación actual del acceso a la información pública.

Centro de Derechos Humanos. (2009)- Informe Anual sobre Derechos Humanos en Chile. Facultad de Derecho. Universidad Diego Portales.

Comisión de Probidad y Transparencia. (2010). Informe 17 Estadístico de Solicitudes. Ley 20.285 sobre Acceso a la Información Pública. Ministerio Secretaría General de la Presidencia.

Comisión Interamericana de Derechos Humanos. (2007). Estudio Especial sobre el Derecho de Acceso a la Información. Relatoría especial para la libertad de expresión. Organización de los Estados Americanos.

(2009). Informe de la Relatoría Especial para la Libertad de Expresión.

Chile Transparente, 2008. Transparencia de Organizaciones No Gubernamentales. Documento de Trabajo ${ }^{\circ}$ 3. Abril 2008.

(2008). Ley de Transparencia de la Función Pública: Garantizando el Acceso a la Información. Documento de trabajo $\mathrm{N}^{\circ} 5$.

(2008). Estándares de Transparencia Municipal. Convenio Asociación Chilena de Municipalidades.

Dazarola, G, s/f: Ley N²0.285 sobre Acceso Información Pública. Área Gobierno, Defensa y Relaciones Internacionales. Asesoría Técnica Parlamentaria, Biblioteca del Congreso Nacional.

Instituto Chileno de Estudios Municipales. Transparencia Activa y Pasiva: Municipios de Chile. Edición 2009-2010. Universidad Autónoma de Chile.

Rehren,A. (2008). La evolución de la agenda de transparencia en los gobiernos de la concertación, Instituto de Ciencia Política, Universidad Católica de Chile.

Rodríguez,A. (2009). Transparencia Activa: Municipios de Chile. Edición mayo-junio 2009. Instituto Chileno de Estudios Municipales. Universidad Autónoma de Chile.

Tello, C.; Cerna, M. y Pavón, A. (2009)- Acceso a la información pública: los desafios del Consejo de la Transparencia. Chile Transparente. Documento de trabajo N8. Citado en Dazarola, G., s/f: Ley N²0.285 sobre Acceso Información Pública. Área Gobierno, Defensa y Relaciones Internacionales. Asesoría Técnica Parlamentaria. Biblioteca del Congreso Nacional.

\section{Sitios electrónicos consultados:}

$<w w w . c h i l e t r a n s p a r e n t e . c l>$

<www.fontra.org>

$<w w w . g o b i e r n o t r a n s p a r e n t e . c l>$

$<w w w . c o n s e j o t r a n s p a r e n c i a . c l>$

<www.transparency.org>

$<w w w$.munitel.cl>

$<w w w$.ciudadanointeligente.cl>

$<w w w . b c n . c l>$

<www.ciperchile.cl>

$<w w w . l e y d e t r a n s p a r e n c i a . c l>$

$<$ www.ichem.cl> 